\title{
ACREDITAR, CERTIFICAR Y EVALUAR Tres procesos distintos y un solo Estado verdadero
}

\begin{abstract}
Resumen
La tesis que aquí se expone sustenta que si la acreditación de las Facultades de Educación es una continuación del proceso de acreditación previa, y si esta última no es una acreditación voluntaria entre pares, sino un mecanismo que el Estado utiliza para otorgar licencias de funcionamiento a los programas, en ejercicio de sus funciones de inspección y vigilancia, es muy probable que esta acreditación se convierta en un mecanismo para evaluar y hacer seguimiento a dichas licencias, y no para cumplir con las funciones de fomento a la calidad. Aquí se analiza esta tendencia del Estado pos-benefactor a través de la cual garantizaría que las universidades le fueran funcionales a un proyecto político e ideológico que parece estarse configurando en el nuevo orden mundial que lidera la economía de mercado.
\end{abstract}

Palabras clave: Acreditación, acreditación voluntaria, acreditación previa, Estado.

\begin{abstract}
The thesis exposed here sustains that if the accreditation of the Abilities of Education is a continuation of the process of previous accreditation, and if this last one is not a voluntary accreditation among even, but a mechanism that the State uses to grant operation licenses to the programs, in exercise of its inspection functions and surveillance, it is very probable that this acreditación becomes a mechanism to evaluate and to make pursuit to this licenses, and it doesn't stop to fulfill the development functions to the quality. Here this tendency of the State searchbenefactor is analyzed through which would guarantee that the universities were functional to a political and ideological project that seems to be configuring in the new world order that leads the market economy.
\end{abstract}

Key words: Accreditation, accreditation voluntary, accreditation previous, State.

\section{Prefacio}

La Ley 30 de 1992 habla del Fomento, de la Inspección y Vigilancia en su capítulo VII. En todos sus artícu- los el énfasis está puesto en el fomento y cuando habla de inspección y vigilancia es para proteger las libertades de enseñanza, aprendizaje, investigación y cátedra, y vigilar que se cumpla la garantía constitucional de la autonomía universitaria;

* Profesor Universidad Pedagógica Nacional - rizoma@andinet.com 
el fomento se refiere a que el Estado debe facilitar el acceso a la ciencia, la técnica y el arte y a la financiación que lo haga viable ${ }^{1}$.

La manera como el CNA ha interpretado sus funciones haría parte de esta tendencia que convierte al Estado en la entidad suprema que define los estándares, esto es, los criterios y los contenidos, de la enseñanza, la investigación y la cátedra, para que a través de procesos de "autoevaluación" la sociedad se informe y controle, a través del libre juego del mercado, a aquellas instituciones y programas que no cumplen los requisitos fijados en dichos estándares. Independientemente de lo que hayan dicho las instancias jurídicas correspondientes, esto es, a todas luces, una flagrante violación del Estado a la autonomía universitaria.

En el caso de las Facultades de Educación, no es comprensible cómo se le hace una excepción al principio constitucional y a la Ley 30 donde establece la autonomía que tienen las universidades para aprobar sus programas. El decreto 272 en ese sentido es producto de una excelente maniobra jurídica digna de todo reconocimiento.

Para demostrar la tesis central es preciso ubicar el contexto internacional en el que surgió esta política de la acreditación de las instituciones y programas de Educación Superior para, desde allí, pone en evidencia cómo en Colombia este proceso ha sido apropiado por el Estado, cuando históricamente ha sido una dinámica propia de las comunidades académicas.

En segundo lugar, hay que caracterizar el proceso que lleva a cabo el Estado colombiano para la acreditación de las instituciones y programas de la Educación Superior, para señalar cómo, además de apropiarse de una dinámica que no le com-

1 Ver el artículo 31 de la Ley 30 de 1992. pete, ha mezclado tres funciones distintas: la evaluación, la certificación y la acreditación, generando confusión, ambigüedad y sobre todo mucha burocracia.

Finalmente, hay que identificar las circunstancias particulares en las que el Estado decidió evaluar y certificar los programas de las Facultades de Educación, en el marco de sus funciones de inspección y vigilancia, para luego valorar el sentido que tendría inscribirse en el proceso de acreditación más general que se lleva a cabo en las instituciones de Educación Superior. Aquí se pondrá en evidencia cómo la acreditación, para el caso de las Facultades de Educación, se convierte, en la práctica, en un mecanismo de certificación.

\section{La tradición internacional muestra que la acreditación es un proceso que le compete a las comunidades académicas y no al Estado}

En los años 70' el tema de la acreditación estaba relacionado con el sistema de créditos que regulaba el tránsito de los estudiantes por un currículo universitario. Los créditos eran la unidad de valor de una asignatura. La acreditación representó entonces un procedimiento encaminado a otorgar reconocimiento a las habilidades y destrezas (tal como se concebían entonces) adquiridas por un estudiante en cada asignatura ${ }^{2}$.

De un proceso de certificación de destrezas individuales se pasó luego a validar la acreditación como un proceso de reconocimiento de planes, programas e instituciones educativas.

\footnotetext{
${ }^{2}$ Secretaría General Ejecutiva de la ANUIES. "Consideraciones generales sobre el proceso de acreditación de las instituciones de educación superior en México". En: Cuadernos ASCUN. Acreditación. No.1. Asociación Colombiana de Universidades. Bogotá, abril de 1995 , p. 5.
}

Otorgar reconocimiento es el sentido que se conserva de aquella concepción que orientó la vida universitaria durante las últimas décadas. Otorgar reconocimiento se ha entendido como la legitimación externa de los resultados de las tareas institucionales. Pero dicho reconocimiento externo lo ha hecho tradicionalmente las comunidades académicas a través de sus distintas formas de organización. Es lo que se conoce como reconocimiento por pares.

En los años 90 se ha generalizado en América Latina la idea de que con este mecanismo se puede mejorar la calidad de la educación superior a partir de la evaluación que de él se desprende. La evaluación sería el diagnóstico que muestra la realidad de lo que se hace en una institución y la acreditación sería la constancia de credibilidad que un organismo externo le da, de acuerdo con estándares de excelencia. Dicha constancia se convierte en una certificación, pero, ¿quién certifica?, ¿quién certifica a quién? En la tradición mundial esto lo han hecho los pares académicos.

Tenemos, entonces, hasta aquí, tres conceptos que se encontraron, aunque venían de procesos distintos: la acreditación, la evaluación y la certificación.

En rigor la acreditación busca establecer una clasificación con base en el grado de adecuación que tiene la institución o el programa frente a los parámetros predefinidos por las comunidades académicas, pero no pretende incidir en la acción y menos en la certificación estatal con la cual se aprueba o no el funcionamiento de un programa o institución.

Acreditar es un asunto que se realiza entre pares y la certificación es el proceso subsiguiente que hace una autoridad competente, creada y regulada por las mismas comunidades académicas, para dar constancia del resultado del proceso de acreditación, esto es, del nivel en el 
que se encuentra un programa frente a los parámetros acordados previamente.

Cuando el Estado intenta utilizar este proceso para autorizar o no licencias de funcionamiento, lo pervierte completamente.

La primera intromisión de un Estado latinoamericano en esta dinámica se presentó en México ${ }^{3}$, cuando se dio cuenta que las instituciones que acreditaban programas no tenían autorización legal, entonces el Estado intervino para regularlas. Sin embargo, lo utilizó para autorizar o no licencias de funcionamiento.

En los países anglosajones, el término acreditación se utiliza en un solo sentido: se emplea para que las asociaciones académicas den su reconocimiento a instituciones y programas.

En EE. UU. la acreditación institucional surgió porque siendo la mayoría de instituciones de educación superior de carácter privado no podían ser intervenidas por el Estado, entonces se crearon asociaciones ad hoc para definir estándares de desarrollo y establecer desde allí el grado de eficacia y eficiencia con el que las instituciones cumplían sus propósitos. Esta preocupación por la eficacia y la eficiencia es muy propia de su cultura. No es una preocupación del Estado, no es una función suya, sino una cultura, esto es, que la población en su mayoría piensa que la eficacia y la eficiencia deben acompañar todas las acciones de la vida social. Allí las instituciones privadas funcionan con ese criterio; es por eso que se autorregulan a través de asociaciones creadas para ese fin, es la sociedad misma la que se ocupa de eso; el Estado puede certificar, pero las instituciones son las que se acreditan entre sí, ese es su problema. Las agencias acreditadoras son

\footnotetext{
${ }^{3}$ Experiencia que ha sido seguida muy de cerca por el CNA colombiano.
}

comunes, su presencia en la vida social está generalizada, aceptada y por lo tanto legitimada, no sólo en el campo de la educación, sino también en el mundo de la empresa, en el de las finanzas, en el comercio, incluso en el medio artístico y cultural. Son las academias las que hacen los reconocimientos sociales, las que premian a los mejores, como una forma de establecer sus propias reglas de producción de valores y de verdades, de validar lo que consideran bueno y sancionar lo que no.

En el caso de la educación, las agencias acreditadoras se han propuesto buscar la continuidad y la coherencia entre los niveles educativos inferiores y los superiores. Existen tres tipos de agencias acreditadoras: la nacionales, que acreditan instituciones, las asociaciones, regionales y los organismos de acreditación de programas académicos específi$\cos ^{4}$.

Este es un modelo de sociedad fundada en la competencia, donde el Estado cumple el papel de arbitro de estos procesos y las organizaciones sociales asumen enteramente la responsabilidad de velar por la idoneidad de sus gestiones. Más allá de emitir un juicio sobre la validez o no de lo que allí sucede, lo cierto es que esto hace parte de una cultura construida a lo largo de cientos de años: ese es su modo de ser social.

Sin embargo, en América Latina hay cierta tendencia a imitar y a adoptar estos esquemas, por efectos de conocidos mecanismos que nos hacen ver dicha cultura como ideal.

Por solicitud expresa de varias instituciones educativas latinoamericanas, especialmente de carácter privado, las asociaciones norteamericanas están ofreciendo sus servicios de acreditación en nuestro mercado académico.

\footnotetext{
${ }^{4}$ Secretaría General Ejecutiva de la ANUIS, op. cit., p. 7.
}

Algunas instituciones educativas de América Latina han solicitado ingreso a las asociaciones internacionales, sometiéndose al cumplimiento pleno de los requisitos que allí se establecen; esto significa que son clasificadas de acuerdo con los estándares de dichas asociaciones, con lo cual esperan ser tenidas en cuenta en sus programas de intercambios, apoyos, etc. Aunque no estén pidiendo que las acrediten, de hecho lo hacen, para poder hacer parte de la asociación y disfrutar de los beneficios que esto trae. En otros casos, lo que se busca es ofrecer programas convalidados internacionalmente, para lo cual se afilian a una asociación que vigila el cumplimiento de los estándares. Es el caso del bachillerato internacional u otros programas de educación técnica o tecnológica que se pueden cursar en uno u otro país a través de diferentes instituciones, dado que han unificado sus planes de estudio. En ciertos casos, para darle prestigio a la universidad o al programa, se le exige a los estudiantes que presenten una prueba de ingreso que corresponde a las exigencias de alguna institución extranjera. Con esto los estudiantes que aprueben pueden tener facilidades de intercambio y su título estaría reconocido por dicha institución extranjera. Esta es otra modalidad de acreditación que tiende a generalizarse. Por lo general, estos programas no tienen su origen en nuestros países latinoa-

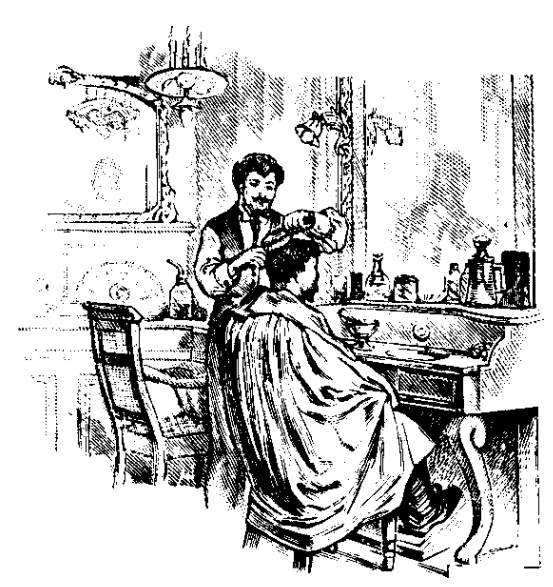


mericanos, la mayoría son norteamericanos y pocos son europeos.

La tendencia en América Latina es crear las asociaciones de profesionales o de facultades para propiciar intercambios y fortalecer el campo específico en el que se mueve cada una de ellas. Para ingresar se establecen una serie de requisitos, proceso que podría asimilarse a la acreditación, pues, de hecho, pertenecer a ellas les da un cierto prestigio. Lo que no se practica es el modelo norteamericano, pues en dichas asociaciones no se acredita a sus afiliados con base en estándares explícitamente elaborados para ese fin.

Sin embargo, en México ya hay experiencias que se acercan al modelo norteamericano; allí las universidades privadas crearon la FIMPES-Federación de Instituciones Mexicanas Particulares de Educación Superior-; para ingresar y permanecer en ella se exige una serie de requisitos y un proceso de evaluación periódica con base en once categorías; entre otras, se hace seguimiento a: la filosofía, propósitos, normas, programas, personal, estudiantes, apoyos, servicios, recursos... cada una con sus respectivos indicadores cuantitativos y cualitativos. Estos parámetros fueron creados por la asociación y se aplican exclusivamente a sus afiliados. La evaluación la hace cada institución con el apoyo de la FIMPES. EI concepto final depende de la información que se entregue y de la visita que le haga un grupo técnico.

El modelo anterior fue tomado de los requisitos que establece la Southern Association of Colleges and Schools-SACS, para la acreditación de instituciones educativas. Estos son:

el procedimiento para la acreditación se inicia con un proceso de autoestudio institucional. Este versa sobre el cuerpo docente, sobre su misión y estructura organizacional, planes y programas de licenciatura y posgrado, servicios de apoyo a la docencia, servicios estudiantiles, administración, investigación y extensión, espacios fí-

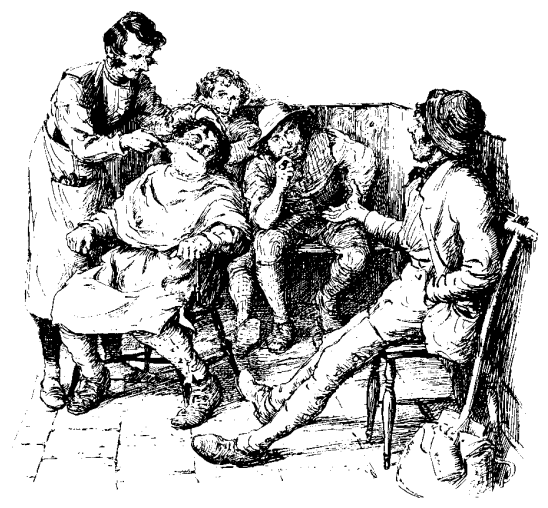

programas de posgrado o los centros de investigación apliquen a la acreditación y dependiendo de su lugar en la clasificación final recibirán o no cierta proporción de los recursos. En Mexico el CONACYT (Consejo Nacional de Ciencia y Tecnología) estableció en sus estándares para la clasificación de programas de investigación, aspectos como: grados académicos de los profesionales, proporción frente a planta administrativa, dedicación, producción científica con arbitraje internacional, líneas de investigación, infraestructura física, vinculación con el sector productivo, entre otros. A partir de estas experiencia el gobierno mexicano creó, en 1989, la Comisión Nacional para la Evaluación de la Educación Superior -CONAEVA- para promover un proceso de evaluación institucional, a realizarse periódicamente. Como vemos, en México, el Estado tiene clara la diferencia entre evaluar, acreditar y certificar.

El principio que guía todos estos procesos es muy sencillo. Hay que apoyar a los mejores; esto supuestamente estimulará a todas las instituciones a hacer las cosas mejor para acceder a dichos recursos. También lo podríamos decir de otra manera: estimulará a las instituciones a hacer las cosas tal como los estándares lo determinan, para poder acceder a los recursos.

Kells H.R, experto en estos temas y asesor del proceso chileno. ha identificado tres modelos de acreditación ${ }^{6}$ :

1. Los mecanismos de revisión de programas, de las universidades canadienses, los sistemas de acreditación en los Estados Unidos y los sistemas de evaluación de México. (...) En dichos sistemas se busca tanto el mejoramiento como la garantía al público, evaluando los programas y la totalidad de la institución y empleando como marco de análisis los propios objetivos y metas planteados por

\footnotetext{
5 Idem, pág. 20. Obsérvese la "coincidencia" con el modelo adoptado por el CNA colombiano, con la diferencia de que aquí los criterios los define y los evalúa el Estado.
}

${ }^{6}$ H.R. Kells. "Ambitos para la regulación". op.cit., p. 32. En: Cuadernos ASCUN. Acreditación. №1, 
las instituciones y las normas escritas y no escritas de los gremios o disciplinas. Ello se lleva a cabo mediante la autoevaluación la que luego se valida por medio de la revisión por un académico o institución equivalente. (...) tienen como finalidad ser sistemas autorreguladores ejecutados por las instituciones y principalmente para beneficio de las mismas. En estos sistemas, donde el poder se ejerce en el nivel más alto o ejecutivo de la universidad, se origina allí un alto grado de regulación, con el fin de tomar decisiones de mercado y anticiparse a toda iniciativa del gobierno para regular. En dichos sistemas las propias instituciones, grupos de instituciones vinculadas en asociaciones regionales y grupos de profesionales evalúan los departamentos o programas y, periódicamente, toda la institución. El objetivo es hacerse presión mutua para mejorar y para dar garantía al público de que esa regulación asegurará el nivel de calidad exigido por los propósitos enunciados por las instituciones y las normas publicadas de los grupos.

2. Sistemas de regulación en el Reino Unido y los países de la Comunidad Británica de Naciones. (...) En dichos sistemas se establecen los objetivos de mantener la equivalencia de grados y prácticas de calificación y se intentan orientar los recursos mediante juicios diferenciales de calidad y la comparación, en el último caso con el uso de indicadores numéricos reduccionistas y comparación normativa y en el primer caso, recurriendo a examinadores externos. Ejemplos serían la práctica de la selectividad en materia de investigación y el inveterado sistema de examinadores externos en el Reino Unido.

3. En varios países de Europa continental (...) los programas universitarios (sólo los aspectos de docencia y de contenido del programa) se revisan más que nada para fines de garantía al público y con algún acento en el mejoramiento, usando principalmente las normas no escritas de los expertos disciplinarios y recurriendo en alto grado a la evaluación por un académico o institución equivalente.

Los sistemas de regulación mencionados se crean especialmente con fines de mejoramiento, pero además tienen el objeto de proteger a los clientes del sistema que carecen de la información, la experiencia, el tiempo o los medios económicos para tomar decisiones críticas en relación con el mercado. Aún más, el sistema de regulación busca garantizar al público que se cumplirán los objetivos enunciados, se satisfarán niveles generalmente aceptados de desempeño y se logrará un grado de eficiencia razonable en el uso de los aranceles cobrados a los usuarios, los impuestos públicos y otros recursos de apoyo.

El mismo autor nos da pistas acerca de los fundamentos que guían estos procesos:

existe en general acuerdo en el sentido de que para administrar cualquier cosa en buena forma, debemos llevar a cabo una secuencia cíclica de etapas: planificación, organización, dotación de personal, motivación o conducción y control (o regulación)... (...) Por lo tanto, la regulación debe ser un aspecto obvio de la vida profesional y de las organizaciones. (...) los clientes lo merecen y a la larga terminarán rebelándose si no es así. Sin una regulación personal y en gran medida informal los profesionales pierden a la larga su orientación idónea y su contacto con las buenas prácticas en su especialidad ${ }^{7}$.

Está claro, pues, que estas dinámicas obedecen a la lógica del mercado y que en estas culturas la producción académica es asimilada a una mercancía cuyos destinatarios, los clientes, tienen derecho a vigilar su proceso de producción, para garantizar que son de calidad. Ahora bien, lo que vigilan ellos es el proceso de acreditación que autónomamente realizan entre sí los productores, en este caso, los académicos que quieren competir en el mercado.

El autor que acabamos de citar también hace una muy importante recomendación:

las autoridades deben entender que los procesos de autorregulación y

\footnotetext{
${ }^{7}$ Op cit. p. 35.
}

racionalización deben en su mayor parte, si no en su totalidad, estar separados. Si el sistema de regulación a largo plazo se considera un plan de reducción nadie querrá participar en él o lo hará de manera mínima y defensiva. Los sistemas orientados al mejoramiento pueden, por supuesto, contemplar sanciones, así como incentivos para estimular las mejoras. No obstante, los sistemas periódicos, grandes, a menudo abruptos para reducir grandes segmentos de programas o de personal deben manejarse separadamente a un alto nivel de la organización, y no se debe pedir a los profesionales de los programas revisarse mutuamente y apuntar el dedo. La actividad de regulación basada en la colaboración debe en su mayor parte ocuparse del mejoramiento de la docencia, el aprendizaje, la investigación, los servicios y el funcionamiento de su unidad de trabajo y de la institución en conjunto ${ }^{8}$.

Esta es una recomendación que más adelante retomaremos para ver cómo el Estado colombiano procedió en el caso de la acreditación previa de las Facultades de Educación.

\section{Acreditar, certificar, evaluar. El caso colombiano}

En contravía de las tendencias internacionales, el Estado colombiano, a través de la Ley 30 de 1992, creó el Sistema Nacional de Acreditación. A su vez, a través del acuerdo 06 de 1995 el Consejo Nacional de Educación Superior, CESU, adopta las políticas generales de acreditación en cuya fundamentación hace explícita tal contrasentido cuando dice:

Lo que justifica en último término la existencia de un Sistema Nacional de Acreditación es el potencial que éste tiene para un mejoramiento de las instituciones y de la educación Superior en general, mayor que el que podría lograr esas mismas instituciones en forma individual, aislada y espontánea?

\footnotetext{
8 Idem, p. 40.

9 Consejo Nacional de Acreditación. Lineamientos para la acreditación. Bogotá: Ministerio de Educación, febrero de 1998, p. 162.
} 
Allí se expresa el principio de la desconfianza sobre el cual se ha montado todo este sistema. Principio que va a chocar, como lo veremos, con la exigencia universal al respeto por la autonomía universitaria. Si se cree que las universidades por sí mismas no son capaces de velar por su mejoramiento, entonces no se cree en su autonomía; en esto no existen medias tintas.

Pero en el párrafo anterior, el acuerdo ha dicho que:

La autonomía y el carácter de servicio público, fundamentos del nuevo orden de la Educación Superior, hacen responsables a las propias instituciones del mejoramiento de su calidad. Sin prejuicio de que el Estado ejerza sus funciones constitucionales de inspección y vigilancia sobre ellas ${ }^{10}$.

Entonces, las instituciones de educación superior son responsables del mejoramiento de su calidad, pero el CNA va a garantizar dicho mejoramiento, en ejercicio de las funciones estatales de inspección y vigilancia. Es todo lo contrario de lo que la Ley 30 le ordena al Estado, cuando, como ya vimos, define la inspección y vigilancia como la obligación que este tiene de garantizar que se respeten las libertades y de fomentar la investigación y el acceso democrático a la ciencia, la técnica y las artes.

Sin embargo, pocos párrafos más adelante el acuerdo dice:

EL CARÁCTER VOLUNTARIO que la Ley 30 de 1992 le da a las instituciones de educación superior de acogerse al Sistema Nacional de acreditación implica que éste es un proceso diferente al de inspección y vigilancia que debe ejercer el Estado, y que, por tanto, la acreditación no reemplaza ${ }^{11}$.

Esta es una de las contradicciones y ambiguedades más graves que posee este sistema. Cuando la vo-

\footnotetext{
10 Ibidem.

${ }^{11}$ Idem, p. 163
}

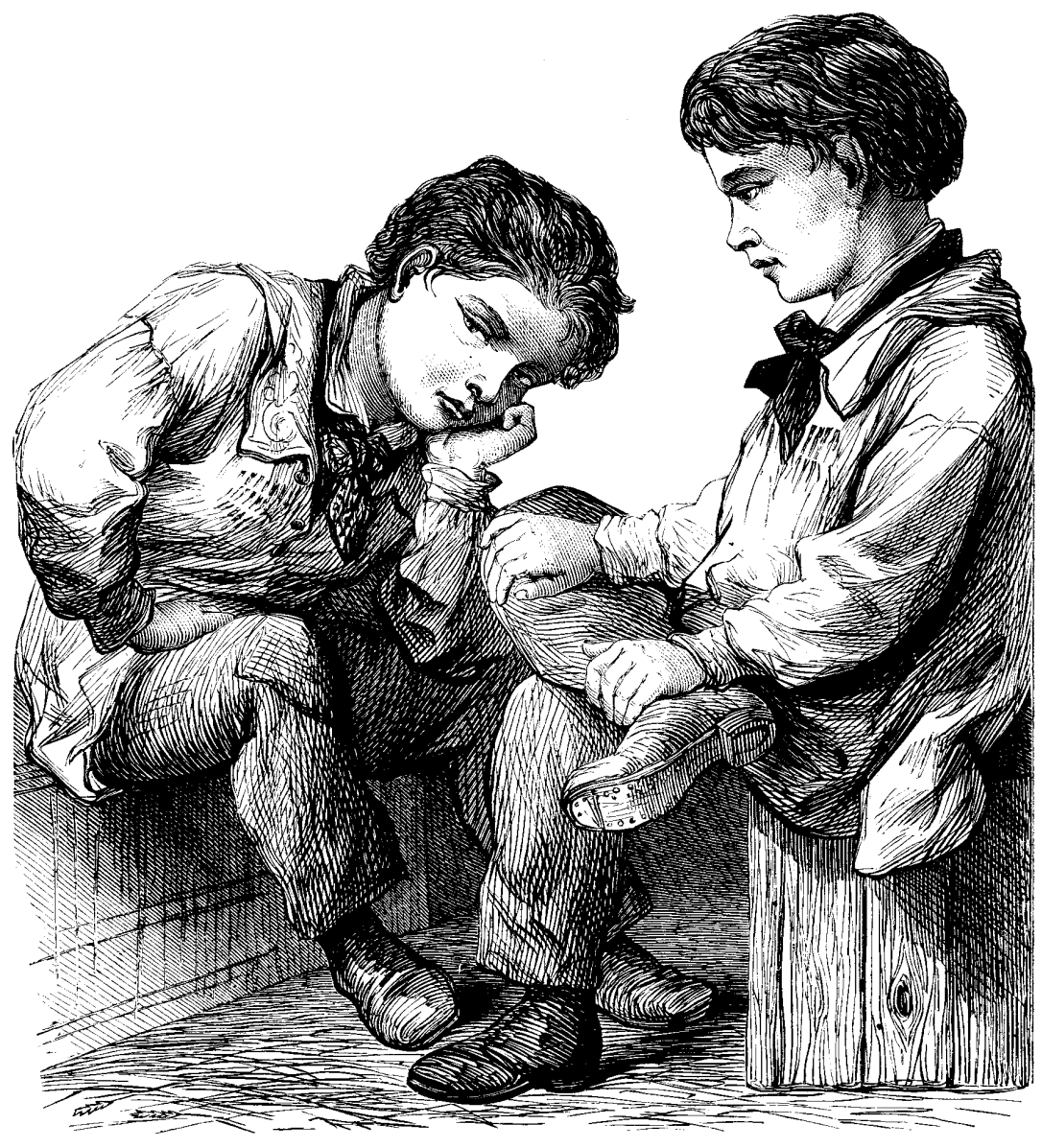

luntariedad la tiene que establecer el Estado a través de una engorrosa legislación, y cuando tiene que advertir en ella que su intervención no corresponde a sus funciones de inspección y vigilancia, es porque algo anda mal. Esta concepción genera una gran ambigüedad: el proceso es voluntario, pero regulado por una Ley que le entrega al Estado una función que en este caso no pretende ejercer.

Con nuestra tradicional Estado intervencionista, esto de la voluntariedad es bastante extraño. Por supuesto que en nuestro contexto el Sistema Nacional de Acreditación es un mecanismo del cual ninguna universidad del país puede marginarse, so pena de desaparecer.

El Estado, aquí se atribuye funciones que en rigor les pertenecen a las comunidades académicas. El Consejo Nacional de Acreditación es quien define qué es la calidad y los criterios para determinarla ${ }^{12}$, pero con el agravante de que introduce una gran confusión. Si estuviéramos discutiendo un documento elaborado por un par académico, el problema sería académico, pero como se trata de una política estatal, la discusión es política, y en política las ambiguedades son muy peligrosas. En primer lugar, define la calidad como la naturaleza de algo; es decir, que la calidad de la educación superior es igual a su naturaleza: una universidad es de calidad si es universidad. En segundo lugar la calidad tiene grado, el más alto corresponde al que se aproxima al tipo ideal que históricamente se define. La

\footnotetext{
${ }^{12}$ Idem, pp. 17-31.
} 
calidad es naturaleza, pero también es histórica, he aquí dos premisas opuestas. Y, ¿quién define el tipo ideal? Siempre hay varios tipos ideales en un momento histórico dado. Como veremos, el tipo ideal lo define el Estado, en el caso colombiano.

Ahora bien, el otro problema, además de las contradicciones y las ambiguedades, es que confunde dos procesos en uno solo: la acreditación y la evaluación de la calidad. Se suponía que la acreditación era simplemente la constancia que el Estado daría del grado de calidad en el que se encontraba una institución de educación superior, de acuerdo con sus estándares. Aquí en Colombia se decidió acreditar programas e instituciones para evaluar su calidad, proceso que se entiende como:

lo que permite reconocer un programa académico específico o una institución de determinado tipo y hacer un juicio sobre la distancia relativa entre el modo como en esa institución o en ese programa académico se presta dicho servicio y el óptimo que corresponde a su naturaleza ${ }^{13}$.

La evaluación es entendida como un juicio, que por supuesto es mucho más que una constancia. Pero si se habla de la naturaleza propia de cada institución, ¿cómo se puede aplicar un mismo proceso de evaluación a un programa y a una institución si son sustancialmente distintos? Y, ¿cómo se pueden comparar dos instituciones o dos programas de naturaleza distinta?

Sin embargo, el Estado es quien define, quíen decide, quien dice cuáles son las características que se deben tener para establecer ese grado de calidad, ese óptimo histórico, esa naturaleza:

Para aproximarse a ese óptimo, el Consejo Nacional de Acreditación ha definido un conjunto de características generales de calidad... ${ }^{14}$.

\footnotetext{
${ }^{13}$ Idem, p. 18.

14 Ibidem.
}

Pero continuando con su ambigüedad, dice que:

Con respecto a ellas se emiten los juicios sobre la calidad de instituciones y programas académicos, pero la determinación más específica y el peso relativo de esas características estarán, en buena parte, condicionadas a la naturaleza de la institución y a la del programa académico en cuestión ${ }^{15}$.

Es una función que se atribuye el Estado pero, de manera vergonzante; además tantas advertencias que hace sobre el respeto a la especificidad de cada institución o programa, es porque sabe, no solamente que viola un principio legal, sino un principio que le es inalienable a las universidades: su autonomía.

Lo que está haciendo el CNA es combinar la creación de estándares universales con la especificidad de cada institución. ¿Cómo puede haber estándares universales sobre aquello que ya se ha definido como histórico y particular? ¿Qué es lo universal? ¿Lo que se hace en América Latina? ¿En Europa? ¿En Estados Unidos? Mientras más grande es el universo, más nos aproximamos a la diversidad infinita.

El CNA, dice también, de manera ambigua, que los universales los establece la misma institución, entonces ¿en qué se constituyen los elementos del proceso de acreditación, las condiciones iniciales para ingresar al sistema, los FACTORES, las características, las variables, los indicadores que suman más de 550 items a cumplir, además de los intrincados procesos de acreditación previa, autoevaluación, evaluación de pares, evaluación final, conceptos, resoluciones, etc.?

Todos estos criterios se constituyen en un rosario infinito de estándares, denominados variables e indicadores que penetran toda, absolutamente toda, la vida universitaria, hasta sus más recónditos rincones.

\footnotetext{
15 Ibidem.
}

Contrario a las recomendaciones internacionales ${ }^{16}$, el CNA pone a los pares académicos a "emitir juicios sobre la calidad"17 como parte del proceso de evaluación externa. El concepto de par académico se pervierte, no sólo por esta razón, sino porque incluye dentro del equipo a especialistas en administración y dirección universitaria, como si fuera lo mismo administrar la química que la educación física, o la filosofía. Lo que demuestra definitivamente que no es una acreditación entre pares sino un juicio, esto es, una certificación que el Estado da a partir de las evaluaciones hechas por los pares, en ejercicio de su función de inspección y vigilancia, entendida a su manera.

Lo que el CNA propone para Colombia es una combinación de los procesos de evaluación de la calidad, certificación de programas e instituciones (otorgamiento de licencias de funcionamiento) y acreditación. Todo esto hecho desde el Estado.

El propósito de todo esto es que las universidades respondan de una manera más eficaz al "desarrollo del país", a las "necesidades del país", a la "modernización y la globalización", a las "demandas del país", como lo dice insistentemente el CNA en la introducción a sus lineamientos. Pero no es difícil saber que no existe un solo país, ni una sola demanda, ni un solo modelo de desarrollo posible, ni una sola vía a la modernización, ni una sola manera de entender la globalización. Pareciera entonces que el propósito es poner en cintura las universidades para que respondan a ciertas exigencias. ¿Dónde quedan entonces la autonomía, y el pensamiento crítico? ¿Dónde queda aquel viejo supuesto de que la universidad es la instancia crítica que toda sociedad democrática necesita y debe cuidar y hacer respetar?

\footnotetext{
${ }^{16}$ Recordemos lo que había señalado el asesor para Chile: consejero Herb. R Kells. 17 Idem, pág. 22.
} 
En vez de obedecer el mandato constitucional que le ordena al Estado fortalecer la investigación científica en las universidades oficiales $y$ privadas y ofrecer condiciones especiales para su desarrollo; en vez de obedecer la función que le asigna la Ley 30 de facilitar mecanismos financieros que hagan posible el acceso de todas las personas aptas a la educación superior, prefiere entender la inspección y vigilancia como un proceso de acreditación, evaluación y certificación, que nos está distrayendo de nuestros objetivos y metas.

\section{Las Facultades de Educación: ¿acreditarse para seguir siendo inspeccionadas? Perspectivas y posibilidades}

Ahora bien, para el caso de las facultades de educación este problema se hace más grave. Aquí el Estado ha asumido funciones excepcionales, por tratarse supuestamente de la formación de profesionales que tienen una alta responsabilidad social. Pero, en realidad, el tema de la formación de maestros se le salió de las manos al Estado y perdió u control. Esto comenzó cuando renunció a la administración y orientación pedagógica de las Escuelas Normales y las dejó convertir en un bachillerato que ofrecía trabajo a los más pobres. Sucedido esto, la formación de maestros quedó en manos de la educación superior, pero las universidades respondieron más a un criterio económico que a una función social. El Estado, en su momento no pudo intervenir este proceso justamente por tratarse de instituciones de educación superior. Cuando se dio cuenta de este problema y de la diversidad de la oferta (propia de una dinámica regulada por la libertad de enseñanza y de cátedra), entonces quiso intervenir, violentando por supuesto el principio de la autonomía universitaria.

El sistema de acreditación le viene como anillo al dedo, porque las puede intervenir bajo el sofisma de la evaluación de la calidad, amparado en el concepto de acreditación, que como vimos es una categoría que le pertenece a las comunidades académicas y no al Estado.

En vez de fomentar el debate, la investigación sistemática y rigurosa y la renovación pedagógica, el Estado ha puesto a las Facultades de Educación a cumplir con un engorroso proceso que no le ha permitido concentrarse en atender la problemática contemporánea de la educación, de la escuela, del maestro y de la pedagogía.

Este proceso de acreditación hay que asociarlo con el decreto 272 que regula los campos de formación. Allí, de nuevo, se irrespeta la autonomía universitaria, y le impide a las facultades confrontarse entre sí para responder al reto de actualizarse y cualificar los programas de formación de maestros, desde la especificidad de cada facultad, de su tradición y de su opción teórica.

En perspectiva, la mejor alternativa sería que las mismas Facultades de Educación asuman las riendas de su propio proceso de cualificación a través de una dinámica académica que hay que inventarse siguiendo nuestra tradición y nuestra cultura ${ }^{18}$. La acreditación por estándares, así sea entre pares, sin que el Estado fije los criterios y las condiciones, es una tendencia importada de la que poco sabemos. Su introducción por la vía autoritaria (legislación estatal), choca en todo caso con nuestras tradiciones y no genera sino resistencias o en el mejor de los casos un agite que distrae por cuanto se hace como cumpliendo la tarea. Esto es lo que nos ha sucedió en realidad. Es muy poco probable que el Estado encuentre allí los datos

\footnotetext{
${ }^{18}$ A nosotros nos gusta discutir, a los sajones no. A nosotros nos gusta encontrarnos, a los sajones no. A los sajones les gustan los estándares, a nosotros no. A los sajones les gustan los resultados medibles y comparables universalmente, a nosotros nos gustan cualitativos, únicos e irrepetibles...
}

que busca; muchos de ellos nos los inventamos.

¿Qué va a pasar con el proceso de acreditación que se inicia después de la acreditación previa? Lo más seguro es que se vuelva a hacer la tarea. También es probable que la experiencia sirva, que se aproveche para que cada facultad se esculque un poco y se encuentre con propuestas de renovación interesantes. Seguramente se dinamizarán procesos, se crearán condiciones para el encuentro y la discusión, se propiciará la autocrítica, en fin, nadie duda que de alguna manera será útil. Sin embargo, todos sabemos que este no es el problema y que además de permitirle al Estado afinar su sistema de información y de control, no se logrará avanzar en la exigente tarea de pensar la educación con seriedad y con altura ética, académica y política.

\section{Bibliografía}

Consejo Nacional de Acreditación. Lineamientos para la acreditación. Ministerio de Educación. Bogotá, febrero de 1998.

Criterios y procedimientos para la acreditación previa de los programas académicos de pregrado y de especialización en educación. Bogotá, junio de 1998.

H.R. Kells: Ambitos para la regulación. En: Cuadernos ASCUN. Acreditación. №1. Asociación Colombiana de Universidades. Bogotá, abril de 1995.

Ministerio de Educación Nacional. Ley 30 del 28 de diciembre de 1992.

PABÓn Fernández, Nohra, et al. Evaluación y Acreditación Universitarias. Bases de Datos y Estado del Arte. Fundación Universitaria de Bogotá Jorge Tadeo Lozano. Bogotá: Universidad Pedagógica Nacional, 1996.

Secretaría General Ejecutiva de la ANUIES. "Consideraciones generales sobre el proceso de acreditación de las instituciones de educación superior en México". En: Cuadernos ASCUN. Acreditación. No.1, Bogotá: Asociación Colombiana de Universidades, abril de 1995. 